\title{
CRITICAL PHENOMENA AT SURFACES AND INTERFACES
}

\author{
REINHARD LIPOWSKY \\ Institut für Festkörperforschung \\ Forschungszentrum Jülich \\ Postfach 1913 \\ D-5170 Jülich \\ FRG
}

\begin{abstract}
The multitude of critical phenomena which occur at surfaces and interfaces is briefly reviewed from a theoretical point of view. Three types of critical effects are distinguished related (i) to the 2-dimensional character of the interface, (ii) to its morphology, and (iii) to its structural changes at phase transitions in the bulk. Wetting phenomena in three dimensions belong to the last category (iii). Some recent theoretical results for such phenomena are also discussed: (a) Wetting of a moving interface; (b) Wetting in the 3-dimensional Ising model; and (c) Wetting of an inhomogeneous substrate.
\end{abstract}

\section{Introduction}

During the last decade, a lot of effort has been devoted to the study of critical phenomena at surfaces and interfaces. Here and below, the term surface denotes the interface between a condensed phase and an inert vapor or 'vacuum'. Since the latter phase is transparent to most experimental probes, many experimental techniques have been used to study surface critical phenomena. However, from a theoretical point of view, there is no fundamental difference between a surface and any other interface between two thermodynamic phases.

One intriguing aspect of interfaces is their reduced dimensionality. Indeed, in many cases, the interface can simply be viewed as a planar 2-dimensional system. However, it can also 'bulge' into the third dimension and then attain nonplanar morphologies. In addition, the interface itself has a third dimension which can become mesoscopic as in wetting phenomena; one then has a system which interpolates between two and three dimensions.

In this paper, I will briefly review the multitude of critical phenomena which can occur at surfaces and interfaces. Three different categories of such phenomena will be distinguished: (i) Critical behavior within the 2-dimensional interface; (ii) Critical effects related to the morphology of interfaces; and (iii) Changes in the interfacial 
structure as one of the adjacent bulk phases undergoes a phase transition.

Wetting processes such as, e.g., surface melting or surface-induced order and disorder belong to the last category (iii). Some recent theoretical results for these processes will also be discussed: (a) Wetting of a moving interface - An example is surface melting of a crystal which slowly evaporates. It is shown that the wetting layer thickens as a result of this motion; (b) Wetting in the 3-dimensional Ising model - In this case, thermally excited shape fluctuations of the two interfaces bounding the wetting layer should be important. Until very recently, computer simulations gave no evidence for such fluctuation effects. It is argued here that the interfacial fluctuations observed in the simulations are presumably spikes rather than smooth deformations; and (c) Wetting of an inhomogeneous substrate - If one of the phases consists of an inhomogeneous solid, the wetting layer experiences a random substrate potential which leads to an increase of the interfacial roughness.

\section{Critical behavior within the 2-dimensional interface}

\subsection{MULTITUDE OF 2-DIMENSIONAL PHASES AND PHASE TRANSITIONS}

Usually, the interface between two 3-dimensional phases has a thickness with is set by the scale of the molecules. It then represents a 2-dimensional subsystem which exhibits a variety of phases and associated phase transitions. Consider, e.g., a monolayer of adatoms adsorbed onto the surface of a 3-dimensional liquid or solid. Such a layer often exhibits a 2-dimensional vapor, liquid, and solid phase. In general, the molecules possess internal degrees of freedom which can order and then lead to additional phases. Examples are the liquid crystalline phases of amphiphilic molecules adsorbed at the water-air interface. If the substrate is a crystal, these 2-dimensional layer phases within the adsorbed layer can be commensurate or incommensurate with this substrate.

The multitude of phases leads to a multitude of critical phenomena. A wellknown example is the critical point of the 2-dimensional vapor liquid coexistence curve; the associated critical behavior should belong to the universality class of the 2-dimensional Ising model as confirmed in many experiments. Critical effects can also occur at discontinuous phase transitions. One example is provided by wetting phenomena such as edge melting which occurs as the 2-dimensional triple point is approached along the solid-vapor coexistence curve, see Fig. 1. In this case, the monolayer contains solid domains surrounded by vapor. As the triple-point temperature is approached, the edges of these domains start to melt and the domain boundaries contain stripes of the 2-dimensional liquid phase.

Another type of transition occurs when a commensurate state of the monolayer is transformed into a (weakly) incommensurate one. The latter state consists of commensurate domains separated by 1 -dimensional domain boundaries. 

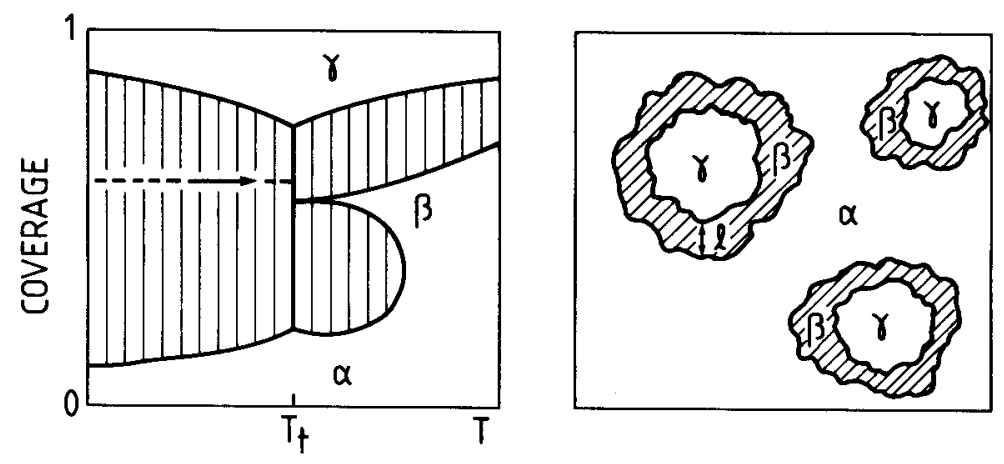

Figure 1: (left) A typical phase diagram for an adsorbed monolayer - The 2dimensional vapor, liquid, and solid phases are denoted by $\alpha, \beta$, and $\gamma$, respectively. When the triple point temperature $T=T_{t}$ is approached along the broken line, the monolayer consists of solid domains, $\gamma$, surrounded by vapor, $\alpha$; (right) Edge melting near the triple point. - The solid domains start to melt along their edges.

The critical effects associated with wetting and commensurate-incommensurate transitions are governed by the behavior of the domain boundaries. This behavior is in itself quite interesting and will be discussed in the next two subsections.

\subsection{ROUGHNESS OF DOMAIN BOUNDARIES}

First, consider a single domain boundary separating two 2-dimensional domains. In equilibrium, the morphology of this domain boundary reflects the underlying symmetry of the system /1/. The following cases have been studied theoretically in some detail: (a) Periodic systems - In this case, the domain boundary is flat or smooth at $T=0$. At $T>0$, it makes transverse excursions $L_{\perp}$ which grow with the longitudinal extension, $L_{\|}$, of the domain boundary. More precisely, one has the scaling law

$$
L_{\perp} \sim L_{\|}^{\zeta}
$$

with the roughness exponent $\zeta=1 / 2$; (b) Quasi-periodic systems - In this case, the behavior in non-universal and depends on the model parameters $/ 2-4 /$. If the domain boundary feels an effective Fibonacci potential, it is rough at all $T$ but the roughness exponent $\zeta$ is $T$-dependent and satisfies $\zeta \leq 1 / 2$; if it feels an effective Harper's potential, the domain boundary undergoes a morphological transition from a smooth state at low $T$ to a rough state at large $T$; and (c) Systems with quenched randomness - In this case, the domain boundary feels a random potential. It then roughens in order to adapt its shape to the random potential and, thus, to minimize 
its energy. As a result, these systems are characterized by an increased roughness exponent: for random field and random bond systems, the scaling law (1) holds again but with $\zeta=1$ (Ref. $/ 5,6 /$ ) and $\zeta=2 / 3$ (Ref. /7,8/) respectively; and (d) Kinetic roughening - Away from equilibrium, growth or shrinkage of the domains implies a moving domain boundary. Various models with local deposition or evaporation rules have recently been studied which lead to a kinetic roughening of the domain boundary $/ 9 /$.

\subsection{FLUCTUATION-INDUCED REPULSION BETWEEN DOMAIN BOUNDARIES}

Next, consider two domain boundaries which are, on average, parallel and have separation, $\ell$. The roughness of these boundaries leads to an effective fluctuation-induced repulsion, $V_{F L}$, with

$$
V_{F L}(\ell) \sim 1 / \ell^{\tau} \quad \text { for large } \ell
$$

where $V_{F L}$ represents a free energy per unit length. Two cases must be distinguished: (i) If the shape fluctuations are thermally excited with $\zeta \leq 1 / 2$, this repulsion arises from a loss of entropy since the configurations of each domain boundary are constrained by the presence of the other one, and

$$
\tau=1 / \zeta \geq 2
$$

as applies to periodic and quasiperiodic systems; /10/ and (ii) For systems with quenched impurities with $\zeta \geq 1 / 2$, on the other hand, this repulsion arises from an increase in energy since each domain boundary cannot explore the minima of the random potential, which lie beyond the other domain boundary, and $/ 11,1 /$

$$
\tau=2(1-\zeta) / \zeta \leq 2
$$

The heuristic concept of a fluctuation-induced interaction just described can be used to determine the correct critical exponents for some wetting phenomena such as edge melting and for commensurate-incommensurate transitions.

Edge melting provides an example for complete wetting. In this case, the wetting layer contains a metastable phase, denoted by $\beta$ in Fig. 1 . Then, in the absence of shape fluctuations, the free energy per unit length of this $\beta$ layer has the generic form

$$
V(\ell)=H \ell+V_{D I}(\ell)
$$

where the pressure--like variable $H$ is proportional to the difference, $f_{\beta}-f_{\alpha \gamma}$, of the bulk free energies of the metastable $\beta$ phase and the stable phases denoted by $\alpha$ and $\gamma$ in Fig. 1. The direct interaction, $V_{D I}(\ell)$, reflects the underlying molecular forces such as van der Waals, electrostatic, or structural forces and decays to zero for large $\ell$. For example, if the adatoms interact via (nonretarded) van der Waals forces, $V_{D I}(\ell) \sim 1 / \ell^{3}$ for large $\ell$. 
Now, the dependence of the mean separation, $\langle\ell\rangle$, on $H$ can simply be obtained by minimization of the free energy $\Delta f(\ell)=H \ell+V_{D I}(\ell)+V_{F L}(\ell)$. Then, two different scaling regimes or universality classes can be distinguished: (i) If $V_{F L}(\ell) \ll V_{D I}(\ell)$ for large $\ell$, the fluctuations do not affect the behavior of $\langle\ell\rangle$ for small $H$; and (ii) If $V_{F L}(\ell) \gg V_{D I}(\ell)$ for large $\ell$, minimization of $\Delta f(\ell) \approx H \ell+V_{F L}(\ell)$ leads to

$$
\langle\ell\rangle \sim 1 / H^{\psi} \quad \text { with } \quad \psi=1 /(1+\tau)
$$

where $\tau$ depends on the roughness exponent $\zeta$ via (3) and (4).

In the latter case, the surface free energy has a singular contribution $f_{s} \simeq H\langle\ell\rangle \sim$ $H^{1-\psi}$ which implies the surface specific heat

$$
c_{s} \sim H^{-\alpha} \quad \text { with } \quad \alpha=1+\psi=(2+\tau) /(1+\tau) .
$$

If the adatoms interact via van der Waals forces and the domain boundaries have the roughness exponent $\zeta=1 / 2$, the relation (7) applies and leads to the specific heat exponent $\alpha=4 / 3$ for edge melting. /12/ Experiments on Neon monolayer gave clear evidence for such a melting process. /13/ The data were fitted with $\alpha \simeq 5 / 4$ which could indicate a crossover effect arising from a relatively large edge stiffness. /1/

A similar line of arguments can be used in order to determine the critical behavior at continuous commensurate-incommensurate transitions. /14/ If $n \sim 1 / \ell$ denotes the density of the domain boundaries, the free energy per unit area, $\Delta F(\ell)$, of the incommensurate state is given by

$$
\Delta F(\ell)=-n \Delta \mu+n\left[V_{D I}(\ell)+V_{F L}(\ell)\right]
$$

where $\Delta \mu$ is the effective chemical potential for the domain boundary. Then, if $\left|V_{D I}(\ell)\right| \ll V_{F L}(\ell)$ for large $\ell$, minimization of $(8)$ with respect to $\ell$ leads to $/ 11 /$

$$
\langle\ell\rangle \sim 1 / \Delta \mu^{\bar{\beta}} \quad \text { with } \quad \bar{\beta}=1 / \tau .
$$

So far, the critical behavior of the domain boundaries has been determined by simple superposition of the direct interaction, $V_{D I}$, and the fluctuation-induced interaction, $V_{F L}$. Such an heuristic approach is, however, not valid in general. One important class of critical phenomena for which this approach fails are wetting transitions for sufficiently-short ranged interactions which satisfy $V_{D I}(\ell) \approx-W / \ell^{\tau}$ for large $\ell$, where $W$ can be negative, zero, or positive. In this case, the interaction is renormalized in a nontrivial way. For periodic systems characterized by $\zeta=1 / 2$ and $\tau=2$, this renormalization can be studied by transfer matrix /15/ and exact functional renormalization group /16/ methods. In this way, a complete classification of wetting transitions in two dimensions has been obtained. /17/ In real systems, such wetting transitions could occur along the steps of vicinal surfaces as has recently been studied in Monte Carlo simulations. /18/ 


\section{Critical effects related to the morphology of interfaces}

\subsection{ROUGHNESS OF INTERFACES}

The morphology of 2-dimensional interfaces in three dimensions is completely analogous to the behavior of 1-dimensional domain boundaries discussed in the previous subsection. Thus, the following cases can again be distinguished: (i) Periodic systems - The surface of a periodic crystal is smooth at sufficiently low $T$ but undergoes a morphological transition to a rough state at a characteristic roughening temperature, $T=T_{R}$. /19/ The roughness is, however, only logarithmic for $T \geq T_{R}$ with $L_{\perp} \sim\left[\ln \left(\left(L_{\|} / a\right)\right]^{1 / 2}\right.$; (ii) Quasi-periodic crystals - The surface of an ideal quasicrystal is predicted to be smooth for all $T / 10,4 /$. This conclusion is based on renormalization group calculations of interface models; (iii) Systems with quenched disorder - In this case, the 2-dimensional interface is presumably always rough with roughness exponent $\zeta>0$. This behavior should also apply to an interface in a random quasicrystal /10/; and (iv) Kinetic roughening - Local deposition rules again lead to an increased roughness of the moving interface and typically to an increased value of $\zeta$. In addition, various morphological transitions between two different rough states of the interface have been predicted $/ 9 /$.

\subsection{EDGE OF A CRYSTAL FACET}

Another aspect of the morphology which is governed by fluctuations is the singular behavior of the equilibrium crystal shape which occurs near the edge of a facet. At such an edge, the facet often meets a rounded part composed of terraces and steps, see Fig. 2. Let $z(x)$ describe this shape: the facet is given by $z(x)=0$ for $x \leq 0$ and the edge of this facet is at $x=0$. Now, the excess free energy of the rounded part arising from the steps can again be estimated as in (8) where $n \sim 1 / \ell \sim d z / d x$ and the effective chemical potential $\Delta \mu \sim x$. It then follows from (9) that $d z / d x \sim x^{\bar{\beta}}$ or $/ 10 /$

$$
z \sim x^{\lambda} \quad \text { with } \quad \lambda=1+1 / \tau
$$

for small $x$ where the exponent $\tau$ as given by (3) or (4) arises from the roughness of the $1-d$ steps. Therefore, the scaling relation $\lambda=1+1 / \tau$ connects the $2 d$ properties of the interface to its $3 d$ morphology. For a periodic crystal, one has $\tau=2$ and thus $\lambda=3 / 2$ as has experimentally been observed, e.g., for small lead crystals. /20/ 


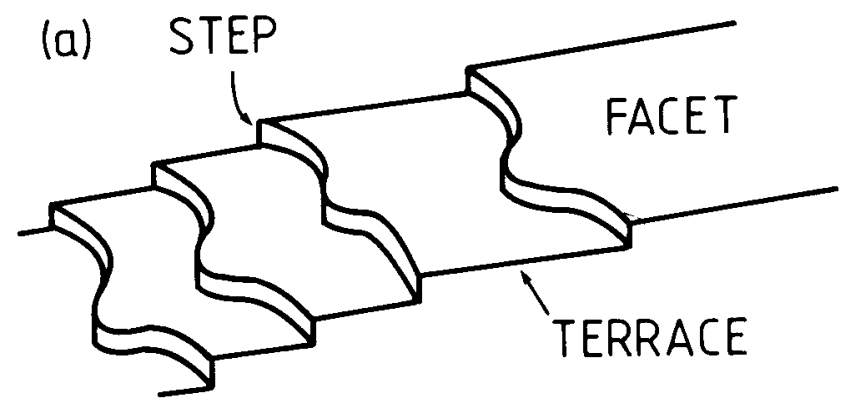

(b)

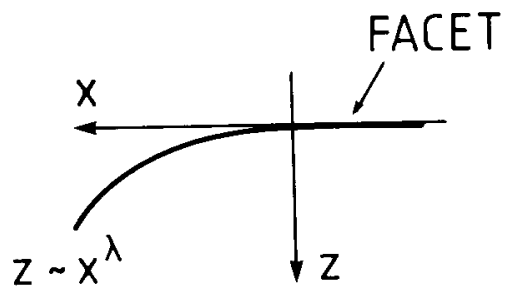

Figure 2: Edge of a crystal facet - (a) On a microscopic scale, the rounded part consists of terraces separated by steps which are, on average parallel to the facet edge; (b) On a macroscopic scale, the rounded part is described by the contour $z(x) \sim x^{\lambda}$ with $\lambda$ as given in (10).

\section{Interfacial structure at phase transformations in the bulk}

When one of the two bulk phases adjacent to the interface undergoes a phase transition, the interface aquires characteristic density profiles. To be more specific, let us consider a crystal-vapor interface, i.e., the surface of a crystal.

\subsection{CONTINUOUS PHASE TRANSITIONS}

The bulk crystal may undergo a variety of continuous phase transitions. If this transition belongs to the universality class of the 3-dimensional Ising model, one can distinguish three types of surface behavior /21-23/: (i) If the coupling constant, $J_{1}$, between two "spins" within the surface is smaller than the coupling constant, $J$, for two "spins" in the bulk, the system undergoes an 'ordinary transition' at the bulk critical temperature $T=T_{c}$ at which both bulk and surface become ordered simultaneously; (ii) If $J_{1}$ is large compared to $J$, the surface undergoes a 'surface transition' at $T_{c 1}>T_{\mathrm{c}}$ while the bulk undergoes an 'extraordinary transition' at 
$T=T_{c}$; and (iii) Finally, for a certain intermediate ratio $J_{1} / J$, bulk and surface simultaneously undergo the so-called 'special transition'.

\subsection{DISCONTINUOUS PHASE TRANSITIONS}

Now, consider again the interface between a crystalline phase, $\gamma$, and a vapor phase, $\alpha$, but assume that the crystal undergoes a discontinuous phase transition between a disordered phase at high temperature and several odered phases at low temperature. Then, a third phase, $\beta$, appears which may prefer to go into the $(\alpha \gamma)$ interface and then forms a thin wetting layer. Two cases may be distinguished: as the transition temperature is approached (i) from below or (ii) from above, a thin layer (i) of the disordered phase or (ii) of one of the ordered phases can be induced by the surface. /24-28/ Alternatively, if both bulk phases $\alpha$ and $\gamma$ are in chemical equilibrium, wetting by the $\beta$ phase may occur as one approaches a triple point at which all three phases $\alpha, \beta$ and $\gamma$ coexist. The latter case is schematically shown in Fig. 3.
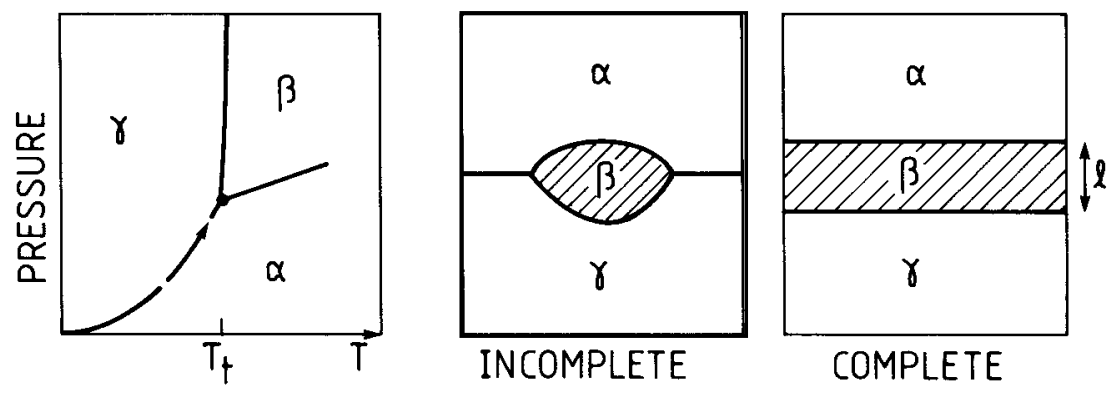

Figure 3: Wetting, surface melting, and related phenomena.- When the $(\alpha \beta \gamma)$ triple point is approached along the $(\alpha \gamma)$ coexistence line (left diagram), the $(\alpha \gamma)$ interface may be (in)completely wet by the $\beta$ phase. Incomplete and complete wetting corresponds to the formation of droplets and a thin wetting layer, respectively. The layer thickness is denoted by $\ell$.

Wetting has to be distinguished from heterogeneous nucleation even though the nucleation barrier is intimately related to the wetting properties. Indeed, heterogeneous nucleation at the interface occurs when the system has passed the triple point and the bulk phases, $\alpha$ and $\gamma$, (or at least one of them) have become thermodynamically unstable. The energy barrier for nucleation of $\beta$ droplets is reduced at 
the interface. For complete wetting, this barrier is, in fact, reduced to zero, and the transformation into the stable $\beta$ phase will start at the interface or surface.

When the $(\alpha \gamma)$ interface contains a wetting layer of $\beta$ phase, it splits up into an $(\alpha \beta)$ and a $(\beta \gamma)$ interface. The separation, $\ell \geq 0$, of these two interfaces is equal to the thickness of the wetting layer. Likewise, the excess free energy, $V(\ell)$, of the layer can be regarded as an effective interaction between these interfaces.

In the absence of interfacial fluctuations, the interaction $V(\ell)$ has the same generic form $V(\ell)=H \ell+V_{D I}(\ell)$ as for domain boundaries, see (5). This interaction is again renormalized by shape fluctuations of the interfaces even though this renormalization is less effective in $d=3$ than in $d=2$. The influence of interfacial fluctuations is studied most conveniently within the framework of effective models for the interfacial separation $\ell \geq 0$. For wetting in three dimensions, these models have the generic form $/ 1 /$

$$
\mathcal{H}\{\ell\}=\int d^{2} x\left\{\frac{1}{2} \bar{\Sigma}(\nabla \ell)^{2}+H \ell+V_{D I}(\ell)\right\}
$$

where $\tilde{\Sigma}$ is an appropriate interfacial stiffness. A hard wall potential at $\ell=0$ which ensures $\ell \geq 0$ is implicitly assumed. In what follows, I will study three different situations which correspond to three different interactions, $V_{D I}(\ell)$.

\subsection{WETTING PHENOMENA IN THREE DIMENSIONS: SOME RECENT THEORETICAL RESULTS}

4.3.1 Wetting away from equilibrium: a moving wetting layer is thicker. First, consider an $(\alpha \gamma)$ interface which moves with constant velocity. An example is a crystal in ultra-high vacuum which slowly evaporates. Now, assume that this interface contains a thin wetting layer. The question is: does the motion of the interface affect the thickness of the layer?

This question can be addressed in the framework of Landau models or density functional theories which lead to density profiles, $M(z, t)$, where $z$ is the coordinate perpendicular to the $(\alpha \gamma)$ interface and $t$ is the time. Let us assume that the motion of the interface is not limited by diffusion. One may then consider a simple relaxational dynamics for the densities $/ 29-31 /$. This leads to solutions $M(z, t)=f(z-v t)$ which move with constant velocity $v$.

The behavior of the densities can be used in order to construct the interaction $V(\ell)$ acting between the $(\alpha \beta)$ and the $(\beta \gamma)$ interface. As a result, one finds a shift of the pressure-like variable $H$ and the direct interaction $/ 30 /$

$$
V_{D I}(\ell)=B \exp \left(-\ell / \ell_{1}\right)+C \exp \left(-\ell / \ell_{2}\right)
$$

where the two length scales are given by

$$
\ell_{1}=\xi_{\beta} /\left(-x+\sqrt{1+x^{2}}\right) \quad \text { and } \quad \ell_{2}=\xi_{\beta} / 2 \sqrt{1+x^{2}}
$$


with $x \equiv v / v_{\beta}$. The parameter $v_{\beta}$ is a velocity scale which depends on the mobility within the $\beta$ layer. The length scale $\xi_{\beta}$ is the bulk correlation length within the $\beta$ phase. If the wetting layer does not move, one has $x=v / v_{\beta}=0$, and $\ell_{1}=\xi_{\beta}=2 \ell_{2}$. In general, the scale $\ell_{1}$ increases with increasing velocity. If one ignores the possible effects of shape fluctuations, the expectation value, $\langle\ell\rangle$, is determined by the minimum of $V_{D I}(\ell)+H \ell$. It then follows that the motion of the interface leads to a thickening of the wetting layer $/ 30 /$.

4.3.2 Wetting in the 3-dimensional Ising model: is it dominated by spikes? Next, consider the Ising model on a simple cubic lattice with a (100) surface. The "spins" interact with nearest-neighbor couplings, $J$, and are subject to a short-ranged surface field. This model exhibits critical wetting transitions which have extensively been studied in Monte Carlo simulations /32/. However, the critical behavior of these transitions is not well-understood. Until very recently, the simulations within the Ising model were thought to be consistent with mean-field theory. In contrast, linearized renormalization group calculations of effective interface models with

$$
V_{D I}(\ell)=B \exp \left(-\ell / \ell_{0}\right)+C \exp \left(-2 \ell / \ell_{0}\right)
$$

predicted nonuniversal critical behavior depending on the parameter $\omega=T / 4 \pi \tilde{\Sigma} \ell_{0}^{2}$ where $\tilde{\Sigma}$ is the interfacial stiffness as in (11). /33,34/

In order to address this controversy, we have studied the solid-on-solid (SOS) limit of the 3-dimensional Ising model by Monte Carlo simulations. /35/ A detailed analysis of the MC data revealed that the SOS model belongs to the same universality class as the Gaussian model given by (11) provided (i) one accepts the results of linear renormalization, and (ii) one makes a proper identification of the model parameters. The reduced stiffness of the SOS model has the value $\tilde{\Sigma} / T=c_{1}(J / T a)^{2}$ with $c_{1} \simeq$ 10.4 , and the direct interaction has the form (14) with $\ell_{0}=\xi=c_{2} a T / J$ and $c_{2} \simeq$ 0.175 . As a consequence, the parameter $\omega=T / 4 \pi \tilde{\Sigma} \ell_{0}^{2}$ has the universal value $\omega \simeq 1 / 4$ for the SOS limit of the 3-dimensional Ising model. /35/

The direct interaction (14) for the Ising model can be derived from mean-field theory for the order parameter density $/ 33,36,37 /$. In this derivation, the length scale $\ell_{0}$ is identical with the bulk correlation length $\xi_{\beta}$ within the $\beta$ layer as follows from (12) and (13) for $x=v / v_{\beta}=0$. The SOS model does not include any bubbles or interfacial overhangs and thus does not contain information about the bulk correlation length. Therefore, the length scale $\xi$ cannot be identical with the bulk correlation length. However, this length scale has another rather direct interpretation in terms of interfacial spikes. Thus, consider two planar interfaces at separation $\ell$ and introduce an interfacial fluctuation which consists simply of a column of reversed spins bridging the gap between the two interfaces. Such a fluctuation has an energy $\triangle E=8 \mathrm{~J} \ell / \mathrm{a}$ and thus a Boltzmann weight $\sim \exp (-8 J \ell / a T)$ ignoring entropic effects. Therefore, these spikes are governed by the length scale $\xi_{s p}=0.125 a \mathrm{~T} / \mathrm{J}$ which is comparable to the scale $\xi$ determined in the simulations. 
Thus, one may distinguish two different types of shape fluctuations of the interface: (i) smooth deformations of the mean-field profile, and (ii) abrupt spikes in the form of thin fingers. In both cases, the critical behavior resulting from the shape fluctuations can be described by an effective Gaussian model as in (11) with a direct interaction as in (14) but with different length scales $\ell_{0}$. The question now is: which type of shape fluctuation is typical for critical wetting in the 3 -dimensional Ising model?

For $J / T=0.35, \xi$ as found in our simulations is about twice as large as the generally accepted value for the bulk correlation length $\xi_{\beta} . / 35 /$ In this case, spikes should dominate and $\omega \simeq 1 / 4$. This conclusion is consistent with a recent analysis of the MC data for the 3-dimensional Ising model $/ 38 /$. This analysis is based on the amplitude ratio for the interfacial susceptibility $\chi_{1}$ and leads to the estimate $\omega \simeq 0.3$ which is rather close to the value $\omega \simeq 1 / 4$ obtained for the SOS model.

4.3.3 Wetting of an inhomogeneous substrate: lateral disorder translates into interfacial roughness. Finally, consider wetting of a smooth solid substrate which has an inhomogeneous composition and thus exerts an effective interaction with a random component. /39/ The corresponding direct interaction is taken to be

$$
V_{D I}(\ell)=V_{R}(\ell)-\left(W+W_{r}\right) v(\ell)
$$

The first term, $V_{R}(\ell)$, and the second term, $-W v(\ell)$ with $W \geq 0$, describe the repulsive and the attractive part of $V_{D I}$, respectively. The third term, $-W_{r} v(\ell)$, corresponds to the random component of $V_{D I}$ where $W_{r}$ is a quenched random variable with zero mean value, $\bar{W}_{r}=0$. The potential $v(\ell)$ is taken to behave as $v(\ell) \sim 1 / \ell^{s}$ for large $\ell$; (non-retarded) van der Waals forces are described by $s=2$.

For complete wetting with $W=0$, the $(\alpha \beta)$ interface unbinds from the substrate as the pressure-like variable $H$ in (5) goes to zero. Alternatively, this interface undergoes a critical wetting transition at $(\alpha \beta)$ coexistence with $H=0$ in the limit of zero $W$. For a relatively thick wetting layer, the interfacial fluctuations are now characterized by $L_{\perp} \sim L_{\|}^{\zeta}$ with $\zeta=1 /(2+s)$ which arises from the random component of the substrate potential. /40/ This increased roughness affects the mean separation $<\ell>$ of the $(\alpha \beta)$ interface from the substrate provided $V_{R}(\ell)$ decays faster than $1 / \ell^{\tau}$ with $\tau=2(1+s)$. In the latter situation, one has $\left\langle\ell>\sim 1 / H^{\psi_{c}}\right.$ with $\psi_{c}=1 /(3+2 s)$ for complete wetting and $\left\langle\ell>\sim 1 / W^{\psi}\right.$ with $\psi=1 /(2+s)$ for critical wetting.

\section{Summary and outlook}

In summary, the multitude of critical phenomena which occur at surfaces and interfaces has briefly been reviewed from the theoretical point of view. These phenomena may be divided up into three large classes: (i) Critical behavior within the interface, i.e., critical phenomena in two dimensions. An example is provided by edge melting, see Fig. 1; (ii) Critical aspects of the interfacial morphology, see, e.g., Fig. 2; and (iii) 
Critical changes in the interfacial structure which are induced by phase transitions in the adjacent bulk phases. If this phase transition is discontinuous, the system can exhibit wetting phenomena, see Fig. 3.

This research field still poses many theoretical challenges. For example, surfaces of ideal quasicrystals have been theoretically predicted to be always smooth. Real quasicrystals, on the other hand, usually contain a certain amount of disorder which acts to roughen these surfaces. /10/ Another area with many open problems is the kinetic roughening of surfaces. A variety of theoretical models with local growth rules has recently been introduced and studied. /9/ These studies should be useful in order to understand real growth of solids from the vapor phase as, e.g., in molecular-beam epitaxy.

On the experimental side, new tools have been recently developed such as surfacesensitive $x$-ray and neutron scattering. /41/ Two different methods have been successfully applied: (i) reflectivity measurements (see, e.g., /42/), and (ii) scattering under total external reflection (see, e.g., /27/). With these methods, one can experimentally study structural changes of the interface on the $n m$ scale such as, e.g., wetting or surface-induced nucleation phenomena.

The surfaces considered in this paper are interfaces between two different bulk phases. Finally, I want to mention another type of surfaces, namely membranes which consist of ultra-thin sheets of molecules. It is interesting to note that these membranes exhibit critical behavior which is rather similar to the critical phenomena described here. /43/

\section{Acknowledgements}

I thank Michael E. Fisher for a helpful discussion about interfacial spikes, and Joanna Cook, Willi Fenzl, Stefan Grotehans, Frank Jülicher, Götz Schmidt, Jörn Sonnenburg, Dietrich Wolf and Joachim Wuttke for stimulating interactions. I also thank the organizers of this conference for their invitation.

\section{References}

/1/ For reviews, see R. Lipowsky, in Random fluctuations and patterns growth, ed. by H.E. Stanley and N. Ostrowsky (Kluwer Academic, Dordrecht 1988) p. 227; G. Forgacs, R. Lipowsky, and T.M. Nieuwenhuizen, to appear in Phase transitions and Critical Phenomena, ed. by C. Domb and J. Lebowitz.

/2/ C. Henley and R. Lipowsky, Phys. Rev. Lett. 59, 1679 (1987).

/3/ A. Garg and D. Levine, Phys. Rev. Lett. 59, 1683 (1987). 
/4/ R. Lipowsky, in Fundamental problems in statistical mechanics VII, ed. by $\mathrm{H}$. van Beijeren (North-Holland, Amsterdam 1990) p. 139, and references therein.

/5/ J. Villain, J. Phys. (Paris) Lett. 43, L551 (1982).

/6/ G. Grinstein and S.-K. Ma, Phys. Rev. B38, 2588 (1983).

/7/ D.A. Huse, C.L. Henley, and D.S. Fisher, Phys. Rev. Lett. 55, 2924 (1985).

/8/ M. Kardar, Phys. Rev. Lett. 55, 2923 (1985).

/9/ For reviews, see D.E. Wolf in Kinetics of ordering and growth at surfaces, ed. by M. Lagally (Plenum Press, New York 1990); and J. Krug and H. Spohn, in Solids far from equilibrium: Growth, morphology and defeats, ed. by C. Godreche (Cambridge University Press, 1991).

/10/ R. Lipowsky and C. Henley, Phys. Rev. Lett. 60, 2394 (1988).

/11/ R. Lipowsky and M.E. Fisher, Phys. Rev. Lett. 56, 472 (1986).

/12/ R. Lipowsky, Phys. Rev. Lett. 52, 1429 (1984); and Phys. Rev. B32, 1731 (1985).

/13/ D.M. Zhu, D. Pengra, and J.G. Dash, Phys. Rev. B37, 5586 (1988).

/14/ For a review, see M.E. Fisher, J. Chem. Soc. Far. Trans. II 82, 1569 (1986).

/15/ R. Lipowsky and T.M. Nieuwenhuizen, J. Phys. A21, L89 (1988); R.K.P. Zia, R. Lipowsky, and D.M. Kroll, Am. J. Phys. 56, 160 (1988).

/16/ F. Jülicher, R. Lipowsky, and H. Müller-Krumbhaar, Europhys. Lett. 11, 657 (1990); H. Spohn, Europhys. Lett. 14, 689 (1991).

/17/ Wetting in 2-dimensional quasiperiodic systems and in 2-dimensional random bond systems has recently been studied by G. Schmidt and R. Lipowsky, to be published, and by J. Wuttke, and R. Lipowsky, submitted to Phys. Rev. B.

/18/ K. Binder, in Kinetics of ordering and growth of surfaces ed. by M. Lagally (Plenum, New York 1990).

/19/ A recent review is in H. van Beijeren and I. Nolden, in Structure and Dynamics of Surfaces II, ed. by W. Schommers and P. van Blanckenhagen (SpringerVerlag, 1987).

/20/ C. Rottmann, M. Wortis, J.C. Heyraud, and J.J. Metois, Phys. Rev. Lett. 52, 1009 (1984). 
/21/ K. Binder, in Phase transitions and critical phenomena, Vol. 8, ed. by C. Domb and J. Lebowitz (Academic Press, London 1983).

/22/ H. Wagner, in Applications of field theory to statistical mechanics, ed. by L. Garrido (Springer, Berlin 1985).

/23/ H.W. Diehl, in Phase transitions and critical phenomena, Vol. 10, ed. by C. Domb and J. Lebowitz (Academic Press, London 1986).

/24/ R. Lipowsky, Phys. Rev. Lett. 49, 1575 (1982); R. Lipowsky and W. Speth, Phys. Rev. B28, 3983 (1983).

/25/ For reviews, see R. Lipowsky, J. Appl. Phys. 55, 2485 (1984); and in Magnetic properties of low-dimensional systems II, ed. by L.M. Falicov, F. Mejia-Lira, and J.L. Moran-Lopez (Springer-Verlag, Berlin 1990) p. 158.

/26/ Surface melting has recently been studied by density functional methods, see R. Ohnesorge, H. Löwen, and H. Wagner, Phys. Rev. A 43, 2870 (1991).

/27/ For a recent x-ray study of surface melting of $A l(110)$, see $\mathrm{H}$. Dosch, T. Höfer, J. Peisel, and R.L. Johnson, Europhys. Lett. 15, 527 (1991).

/28/ Surface--induced order in $f c c$ alloys has recently been studied by W. Schweika, K. Binder, and D.P. Landau, Phys. Rev. Lett. 65, 3321 (1990).

/29/ T. Meister and H. Müller-Krumbhaar, Z. Physik B55, 111 (1984).

/30/ H. Löwen and R. Lipowsky, Phys. Rev. B43, 3507 (1991).

/31/ Diffusion-limited growth of wetting layers is studied in R. Lipowsky and D.A. Huse, Phys. Rev. Lett. 57, 353 (1986).

/32/ K. Binder and D.P. Landau, Phys. Rev. B37, 1745 (1988).

/33/ R. Lipowsky, D.M. Kroll, and R.K.P. Zia, Phys. Rev. B27, 4499 (1983) used a variational method which is equivalent to linear renormalization. Their parameter $\tau$ corresponds to $[4 \pi \omega]^{1 / 2}$.

/34/ E. Brezin, B. Halperin, and S. Leibler, Phys. Rev. Lett. 50, 1387 (1983); D.S. Fisher and D.A. Huse, Phys. Rev. B32, 247 (1985).

/35/ G. Gompper, D.M. Kroll, and R. Lipowsky, Phys. Rev. B42, 961 (1990).

/36/ E. Brezin, B. Halperin, and S. Leibler, J. Phys. (Paris) 44, 775 (1983).

/37/ M.E. Fisher and A.J. Jin, University of Maryland, preprint.

/38/ A.O. Parry, R. Evans, and K. Binder, University of Mainz, preprint. 
/39/ This situation has to be distinguished from wetting in 3-d random systems, see R. Lipowsky and M.E. Fisher, Phys. Rev. Lett. 56, 472 (1986), and from wetting of a rough solid substrate, see M. Kardar and J. Indekeu, Europhys. Lett. 12, 161 (1990); H. Li and M. Kardar, Phys. Rev. B42, 6546 (1990).

/40/ For a $d_{\|}$-dimensional interface in $d=d_{\|}+1$, one has $\zeta=(5-d) / 2(2+s)$ which exceeds the thermally-excited roughness for $s<s^{*}$ with $s^{*}=(d-1) /(3-d)$ for $1<d \leq 3$ and $s^{*}=\infty$ for $3<d \leq 5$. If $s<s^{*}$ and $V_{R}(\ell) \ll 1 / \ell^{\tau}$ with $\tau=2(d-1+2 s) /(5-d)$, complete and critical wetting are characterized by $\psi=(5-d) /(d+3+4 s)$ and $\psi=(5-d) /(d-1)(2+s)$, respectively.

/41/ See, e.g., Surface x-ray and neutron scattering, ed. by H. Zabel and I.K. Robinson (Springer Verlag, to be published)

/42/ C. Cevc, W. Fenzl, and L. Sigl, Science 249, 1161 (1990)

/43/ R. Lipowsky, Nature 349, 475 (1991) 